\title{
A System and Method for Non-Invasive Measurement of Cardiovascular Blood Pressure
}

\author{
Dr. Alexander Brenner*, Felix Brenner, Yuri Brodsky, David Fiks, Sergey Yusimov, Prof. Avraham Lorber \\ Pi - Harvest Israel Ltd, Bar Yehuda 53, Nesher, Israel \\ Received: 跙 July 12, 2018; Published: 阱July 24, 2018 \\ *Corresponding author: Alexander Brenner, Pi - Harvest Israel Ltd, Bar Yehuda 53, 3688312, Nesher, Israel, \\ Email: alexander.brenner@pi-harvest.com
}

\begin{abstract}
A system and method for the non-invasive portable ultrasound-based measurement system of the Cardiovascular blood pressure is presented, wherein the blood pressure measurement model is calibrated to the simultaneous direct measurements by pressure transducers during the catheterization. The model is based on the image time series processing estimating the changes of the oscillating traceable regions via the introduction of the new ultrasound generalized M-Mode and the notion of the derived image. Once calibrated the system is ready to recalculate the pressure in the calibrated region, such as Left Atrium according to the subsequent non-invasive ultrasound measurements. The system has been successfully tested on animals (sheep) under subsequent catheterisations.
\end{abstract}

Abbreviations: CHF: Congestive Heart Failure; LAP: Left Atrium Pressure; RAP: Right Atrium Pressure; LVEDP: Left Ventricular End Diastolic Pressure; PAP: Pulmonary Artery Pressure; PCWP: Pulmonary Capillary Wedge Pressure; AFR: Atrial Flow Regulator; ICP: Intra-Cardiac (blood) Pressure; US: Ultrasound.

\section{Introduction}

Diseases including CHF is a major cause of premature death: according to the American Heart Association, one in 8 deaths has CHF mentioned on the death [1]. Measuring PAP and LAP (or its proxy PCWP) directly helps to predict CHF as pulmonary hypertension is a major chronic disease associated with CHF [2]. LAP could serve as marker for left ventricle diastolic dysfunction, which is highly connected to heart failure, if it would not be shown that it is not exactly equivalent to Left Ventricular End Diastolic Pressure (LVEDP) [3]. Currently used implantable devices allow home environment measurements and can provide reliable pressure (mainly PAP), but their usage is limited and requires at least the catheterization. The last is an invasive procedure but should not necessarily result in any implantations or on the contrary can serve for Pacemaker or Occluder devices implantations. The proposed system and method utilise any catheterization procedure and allow home environment measurements and monitoring of the CHF susceptible patients.

\section{Method}

The currentapproach is based on the synchronized simultaneous measurements of the ICP with the micro-manometer catheters (pressure transducers) and US recordings with the subsequent signal and image processing. The animal trials were carried out under the approval of the Helsinki research ethics committee: during the first catheterization of each 7 months' age lamb (50$60 \mathrm{~kg}$ ) a device was implanted into the inter-atrial septum: one sheep has got AFR (from Occlutech International AB) having balltipped $3 \mathrm{~mm}$ width $15 \mathrm{~mm}$ length nitinol plate (Figure 1a), second same size regular AFR (Figure 1b) and the third - Occlutech Figulla Flex II PFO occluder (Figure 1c). Every device implantation was proceeding under fluoroscopy and intra-cardiac echocardiography (ICE). The ICP was measured on device implantation and then after 3 and 6 months. At each procedure the sheep was anesthetized and breathed with the lung ventilation machine. LA, RA, LV, RV, PA and PAW pressures were measured by catheters simultaneously with US from the left side of lamb's chest. The final catheterizations additionally contained the measurements under Phenylephrine/ Adrenaline injections to increase ICPs. The overall scheme of the calibration system is shown in (Figure 2).

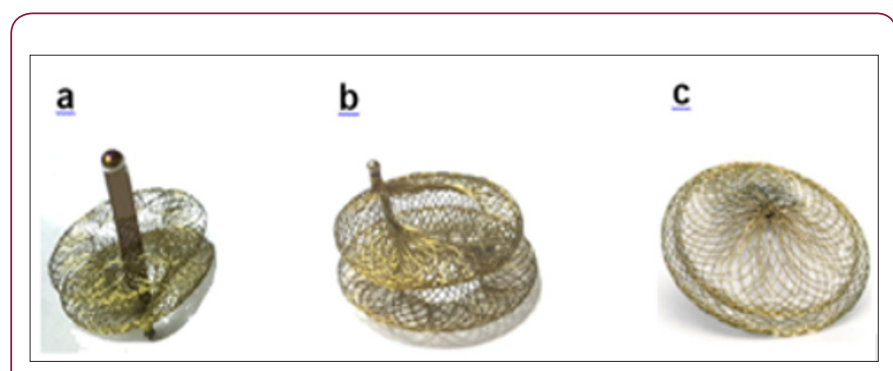

Figure 1: Ball-tipped 3mm width $15 \mathrm{~mm}$ length nitinol plate (Figure 1a), second - same size regular AFR (Figure 1b) and the third - Occlutech Figulla Flex II PFO occluder (Figure 1c). 


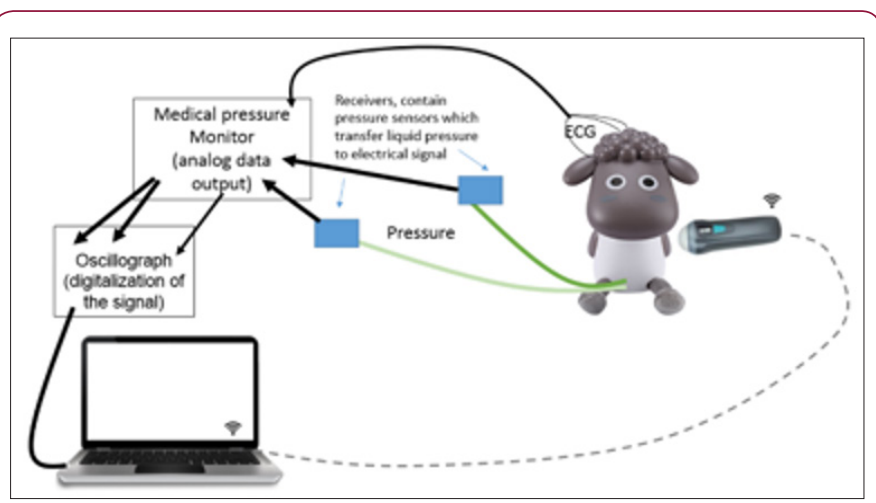

Figure 2: The overall scheme of the calibration system.

The background of the current method of the recorded data analysis is based on the work [4], where it was stated that the waveforms of LA volume and simultaneous LA pressure are similar. This leads to the conjecture that the changes of LA, RA,... pressure $\mathbf{P}$ can be modelled as a function $\mathrm{F}$ of the changes of LA,RA,... volume $\mathbf{V}$ : $\mathbf{P}(\mathrm{t})=\mathrm{F}(\mathbf{V}(\mathrm{t}))$. To estimate the function $\mathrm{F}$ we analyse the series of the US images and calibrate them to the synchronized recording of the respective pressure transducer. The recording time of about 50 sec (at $12 \mathrm{f} / \mathrm{sec}$ US speed) is sufficient to subsequently reproduce the pressure ICP of the same patient using US only. To analyse the US data we introduce the generalized M-mode being the set of M-modes corresponding to all initially formed US beams, retrieving the signal changes for a number of seconds. Typically this is the $3 \mathrm{D}$ array of brightness values $\left\{\mathrm{J}_{\mathrm{i}}\right\}_{\mathrm{i}=1, \ldots \mathrm{N}}$ in polar coordinates over the time. Compressing each frame into a single depth column we produce the derived image $\left\{\mathrm{I}_{\mathrm{i}}\right\}_{\mathrm{i}=1, \ldots \mathrm{N}}$ defined as a chronological union of the averages of the rows or other invariants as singular values of the grey-scaled images $\left\{\mathrm{G}_{\mathrm{i}}\right\}_{\mathrm{i}=1, \ldots \mathrm{N}}$ corresponding to the initial generalized M-mode series $\left\{\mathrm{J}_{\mathrm{i}}\right\}$ across each given depth. Calculation of a local pressure $\mathbf{P}$ at time moments $t$ is reduced to evaluation a function $\mathbf{P} \approx \mathrm{F}\left(\mathrm{L}_{1}, \mathrm{~L}_{2}, \ldots, \mathrm{L}_{\mathrm{n}}\right)$ of the is the most pronounced brightness lines $\mathrm{L}_{1}, \mathrm{~L}_{2}, \ldots, \mathrm{L}_{\mathrm{n}}$ and is based on the calibration fit process to the measurements $\mathrm{P}_{\mathrm{i}}$ at time moments $\mathrm{t}_{\mathrm{i}}$ of $\mathrm{P}_{\mathrm{i}}$ from $\mathrm{L}_{1 \mathrm{i}}, \mathrm{L}_{2 \mathrm{i}}, \ldots, \mathrm{L}_{\mathrm{ni}}$ for the prescribed shape of the function $F$. In case we wish to examine a small bounded volume most pronounced brightness lines can be removed by consequent ones. For linear function $\mathrm{F}$ of the brightness lines $\mathrm{L}_{1}, \mathrm{~L}_{2}, \ldots$ , $\mathrm{L}_{\mathrm{n}}$ with the coefficients $\mathrm{W}_{1}, \mathrm{~W}_{2}, \ldots, \mathrm{W}_{\mathrm{n}}$ optimally fitted for equations $\mathrm{P}_{\mathrm{i}} \approx \mathrm{W}_{1} \mathrm{~L}_{1 \mathrm{i}}+\cdots+\mathrm{W}_{\mathrm{n}} \mathrm{L}_{\mathrm{ni}}+\mathrm{C}$ to hold for subsequent ultrasound recordings one gets the coefficients $\mathrm{W}_{1}, \mathrm{~W}_{2}, \ldots, \mathrm{W}_{\mathrm{n}}$ proportional to the cut areas of the target volume at given depths orthogonally to the transducer working plane, while the whole sum is approximating the pressure as the function of the target area volume according to the Simpson formula as the volume can be represented as $\mathbf{V}\left(\mathrm{t}_{\mathrm{i}}\right)=\tilde{W}_{1} \mathrm{~L}_{1 \mathrm{i}}+\cdots+\tilde{W}_{\mathrm{n}} \mathrm{L}_{\mathrm{ni}}$ , where $\tilde{W}_{i}$ are the real cut areas of the target volume at given depths orthogonally to the transducer working plane and $\mathrm{L}_{\mathrm{ji}}$ indicate the height of the slices. The procedure of the best fit to the measured data provides the accurate hypothesis for the weights $\mathrm{W}_{1}, \mathrm{~W}_{2}, \ldots, \mathrm{W}_{\mathrm{n}}$, C. The weights are stored for further pressure calculations based on US estimations of $\mathrm{L}_{1 \mathrm{i}}, \mathrm{L}_{2 \mathrm{i}}, \ldots, \mathrm{L}_{\mathrm{ni}}$ from subsequent recordings.

\section{Results}

The model proved to be very accurate - the differences between calculated and actual measurements are less than $1.5 \mathrm{mmHg}$ on average for both RA, LA and PAW pressures, see the attached Tables $1-3$. In the tables each cell $(i, j)$ contains the model calibrated to experiment $i$ measurement and further calculated and compared to the actual measurement of experiment $j$ (Figure 3). The results prove that the system is capable to assess the LA, RA, LV, RV, PA and PAW pressures in sheep after the calibration for 6 months and with up to $\pm 40 \%$ weight change (Tables $1-3$ ). The model properly reflects the pressure changes caused by the lung ventilation machine and Phenylephrine/ Adrenaline injections and is indifferent to presence of implants (Figure 1).

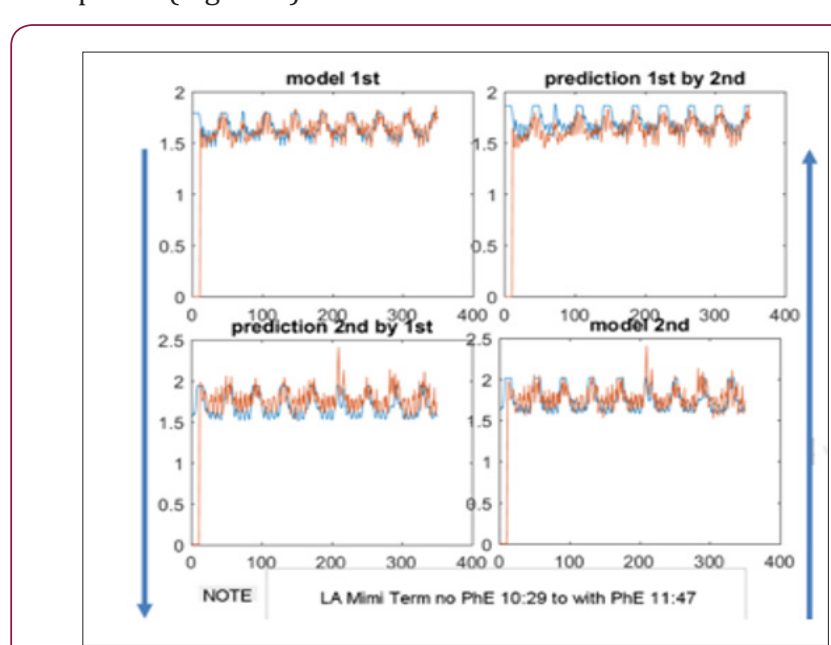

Figure 3: The model calibrated to experiment $i=1,2$ measurement and further calculated and compared to the actual measurement of experiment $\mathrm{j}=2,1$.

Table 1: The results prove that the system is capable to assess the LA, RA, LV, RV, PA and PAW pressures in sheep after the calibration for 6 months and with up to $\pm 40 \%$ weight change

\begin{tabular}{|c|c|c|c|c|c|c|c|}
\hline Mimi RA & $\mathbf{1 - 1}$ & $\mathbf{1 - 2}$ & $\mathbf{1 - 3}$ & $\mathbf{1 - 4}$ & $\mathbf{2 - 1}$ & $\mathbf{2 - 2}$ & $\mathbf{2 - 3}$ \\
\hline $1-1$ & 0.00 & 0.05 & 0.14 & 0.70 & 0.18 & 1.09 & 0.41 \\
\hline $1-2$ & 0.05 & 0.00 & 0.09 & 0.75 & 0.23 & 1.15 & 0.46 \\
\hline $1-3$ & 0.14 & 0.09 & 0.00 & 0.84 & 0.34 & 1.26 & 0.57 \\
\hline $1-4$ & 0.70 & 0.74 & 0.84 & 0.00 & 0.59 & 0.26 & 0.36 \\
\hline $2-1$ & 0.16 & 0.21 & 0.30 & 0.54 & 0.00 & 0.90 & 0.23 \\
\hline $2-2$ & 0.92 & 0.95 & 1.06 & 0.22 & 0.84 & 0.00 & 0.60 \\
\hline $2-3$ & 0.37 & 0.42 & 0.51 & 0.33 & 0.23 & 0.65 & 0.00 \\
\hline
\end{tabular}

Table 2.

\begin{tabular}{|c|c|c|c|c|c|c|c|}
\hline Mimi LA & $\mathbf{2 - 1}$ & $\mathbf{2 - 2}$ & $\mathbf{2 - 3}$ & $\mathbf{2}-\mathbf{4}$ & $\mathbf{2}-\mathbf{5}$ & $\mathbf{2}-\mathbf{6}$ & $\mathbf{2}-\mathbf{7}$ \\
\hline $2-1$ & 0.00 & 0.25 & 0.34 & 0.24 & 0.86 & 0.51 & 0.48 \\
\hline $2-2$ & 0.23 & 0.00 & 0.11 & 0.02 & 0.60 & 0.25 & 0.23 \\
\hline $2-3$ & 0.34 & 0.11 & 0.00 & 0.14 & 0.48 & 0.13 & 0.11 \\
\hline $2-4$ & 0.21 & 0.02 & 0.13 & 0.00 & 0.63 & 0.27 & 0.25 \\
\hline $2-5$ & 0.78 & 0.58 & 0.43 & 0.63 & 0.00 & 0.36 & 0.37 \\
\hline $2-6$ & 0.45 & 0.24 & 0.11 & 0.27 & 0.36 & 0.00 & 0.02 \\
\hline $2-7$ & 0.44 & 0.22 & 0.10 & 0.25 & 0.37 & 0.02 & 0.00 \\
\hline
\end{tabular}


Table 3.

\begin{tabular}{|c|c|c|c|c|c|c|c|}
\hline Ayumi LA & $1-1$ & $1-2$ & $1-3$ & $1-4$ & $1-5$ & $1-6$ & $1-7$ \\
\hline $1-1$ & 0.00 & 0.29 & 0.27 & 0.27 & 0.31 & 0.30 & 0.35 \\
\hline $1-2$ & 0.25 & 0.00 & 0.04 & 0.04 & 0.05 & 0.00 & 0.08 \\
\hline $1-3$ & 0.73 & 0.56 & 0.00 & 0.42 & 0.44 & 0.57 & 0.44 \\
\hline $1-4$ & 0.29 & 0.05 & 0.00 & 0.00 & 0.01 & 0.05 & 0.04 \\
\hline $1-5$ & 0.31 & 0.06 & 0.01 & 0.01 & 0.00 & 0.06 & 0.03 \\
\hline $1-6$ & 0.25 & 0.00 & 0.04 & 0.04 & 0.05 & 0.00 & 0.08 \\
\hline $1-7$ & 0.33 & 0.09 & 0.04 & 0.04 & 0.02 & 0.09 & 0.00 \\
\hline
\end{tabular}

Table 4.

\begin{tabular}{|c|c|c|c|c|}
\hline Mimi LA PAW & PAW - 1 & PAW - 2 & LA - 1 & LA - 2 \\
\hline PAW - 1 & 0.00 & 0.15 & 0.02 & 0.21 \\
\hline PAW - 2 & 0.15 & 0.00 & 0.13 & 0.06 \\
\hline LA - 1 & 0.02 & 0.13 & 0.00 & 0.19 \\
\hline LA - 2 & 0.22 & 0.07 & 0.19 & 0.00 \\
\hline
\end{tabular}

\section{Discussion}

Based on the current approach the human heart chamber pressures can be accessed in the similar way. While the sheep anatomy permits the side view only (analogues of the Apical view), in humans the model has to be adjusted for each of the views. LVEDP can be easily assessed both during the calibrations (especially when having synchronous ECG in an additional channel) either from LA or PAW recordings and from the model during subsequent US analysis. The PAW model accurately predicts LA and vice versa as was expected (Table 4).

\section{References}

1. Benjamin, Michael J Blaha, Stephanie E Chiuve, Mary Cushman, Sandeep R Das, et al. (2017) Heart disease and stroke statistics 2017 update: a report from the American Heart Association. Circulation 138(2): 378393.

2. Mooney DM, Fung E, Doshi RN, Shavelle DM (2015) Evolution from electrophysiologic to hemodynamic monitoring: the story of left atrial and pulmonary artery pressure monitors. Front Physiol 6:271.

3. Nerlekar N, Peverill RE (2018) Confusion regarding the meaning of the term left ventricular filling pressure given the non-equivalence of left ventricular end-diastolic pressure and mean left atrial pressure. Am Heart J 196: e1-e2.

4. Stefanadis C, Dernellis J, Toutouzas P (2001) A clinical appraisal of left atrial function. European Heart Journal 22: 22-36.
ISSN: 2574-1241

DOI: 10.26717/BJSTR.2018.07.001466

Alexander Brenner. Biomed J Sci \& Tech Res

(c) (i) This work is licensed under Creative Submission Link: https://biomedres.us/submit-manuscript.php
Commons Attribution 4.0 License

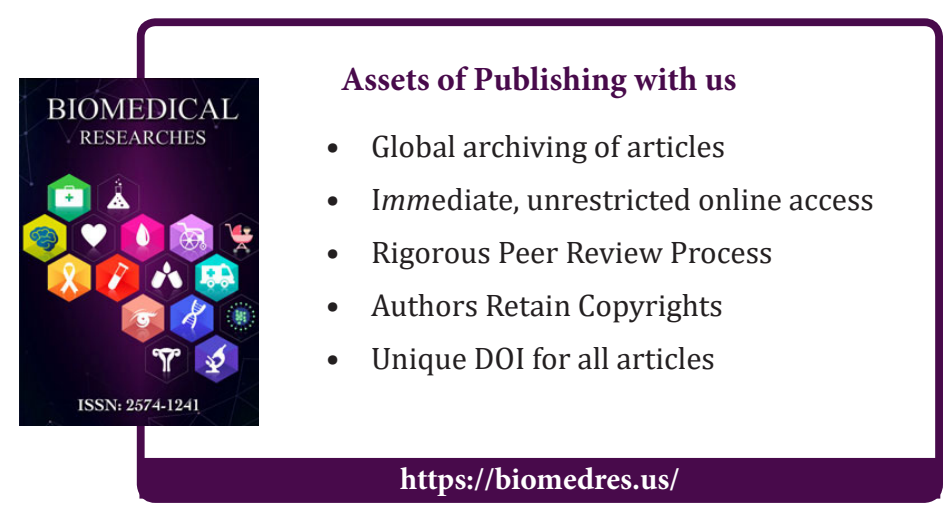

